\title{
On the inviscid limit of the 2D Navier-Stokes equations with vorticity belonging to BMO-type spaces
}

\author{
Frédéric Bernicot ${ }^{\mathrm{a}, *}$, Tarek Elgindi ${ }^{\mathrm{b}}$, Sahbi Keraani ${ }^{\mathrm{c}}$ \\ ${ }^{a}$ CNRS - Université de Nantes, Laboratoire de Mathématiques Jean Leray, 2, Rue de la Houssinière, 44322 Nantes Cedex 03, France \\ ${ }^{\mathrm{b}}$ Courant Institute of Mathematical Sciences, 251 Mercer Street, New York, 10012-1185 NY, USA \\ ${ }^{\mathrm{c}}$ UFR de Mathématiques, Université de Lille 1, 59655 Villeneuve d'Ascq Cedex, France
}

Received 22 January 2014; received in revised form 26 November 2014; accepted 11 December 2014

Available online 13 January 2015

\begin{abstract}
In a recent paper [6], the global well-posedness of the two-dimensional Euler equation with vorticity in $L^{1} \cap \mathrm{LBMO}$ was proved, where LBMO is a Banach space which is strictly imbricated between $L^{\infty}$ and BMO. In the present paper we prove a global result on the inviscid limit of the Navier-Stokes system with data in this space and other spaces with the same BMO flavor. Some results of local uniform estimates on solutions of the Navier-Stokes equations, independent of the viscosity, are also obtained.

(c) 2015 L'Association Publications de l'Institut Henri Poincaré. Published by Elsevier B.V. All rights reserved.
\end{abstract}

MSC: 76B03; 35Q35

Keywords: 2D incompressible Navier-Stokes equations; Inviscid limit; Global well-posedness; BMO-type space

\section{Introduction}

In this work, we consider the problem of the inviscid limit of the 2D Navier-Stokes equations with rough initial data. More precisely, we are interested in the situation where the vorticity lives in specific Morrey-Campanato spaces (in the same flavor as already studied in [6,4] and very recently in [12]). Morrey-Campanato spaces are Banach spaces which extend the notion of a BMO function (a function with bounded mean oscillation) describing situations where the oscillation of the function in a ball is controlled depending upon the radius of the ball. These spaces have attracted much attention in the last few decades due to their remarkable properties (John-Nirenberg inequalities, duality with Hardy spaces, etc.). For example, the theory of Morrey-Campanato spaces is useful when the Sobolev embedding theorem is not available and has proven to be particularly useful in the study of elliptic PDEs.

\footnotetext{
This work was partially supported by the ANR under the project AFoMEN No. 2011-JS01-001-01 and by the NSF Grants DMS-1211806 and DMS-0807347.

* Corresponding author.

E-mail addresses: frederic.bernicot@univ-nantes.fr (F. Bernicot), elgindi@cims.nyu.edu (T. Elgindi), sahbi.keraani@univ-lille1.fr

(S. Keraani).
} 
We do not detail the literature about these spaces since it is huge. In this work, we only focus on the $L^{\alpha} m o$ spaces (see precise definitions in Section 2) where the oscillations of a function on a ball of radius $r \ll 1$ are bounded by $|\log (r)|^{-\alpha}$. What is interesting, is that the scale $\left(L^{\alpha} m o\right)_{0<\alpha<1}$ can be thought of as an intermediate scale between BMO (for $\alpha \rightarrow 0$ ) and $L^{\infty}$ (for $\alpha \rightarrow 1$ ).

\subsection{The Navier-Stokes system}

The Navier-Stokes system is the basic mathematical model for viscous incompressible flows and reads as follows:

$$
\left(N S_{\varepsilon}\right) \quad\left\{\begin{array}{l}
\partial_{t} u^{\varepsilon}+u^{\varepsilon} \cdot \nabla u^{\varepsilon}-\varepsilon \Delta u^{\varepsilon}+\nabla P^{\varepsilon}=0, \\
\nabla \cdot u^{\varepsilon}=0, \\
u_{\mid t=0}^{\varepsilon}=u_{0} .
\end{array}\right.
$$

Associated to the viscosity parameter $\varepsilon$, the vector field $u^{\varepsilon}$ stands for the velocity of the fluid, the quantity $P^{\varepsilon}$ denotes the scalar pressure, and $\nabla \cdot u^{\varepsilon}=0$ means that the fluid is incompressible. We also detail the fractional Navier-Stokes equation, of order $\alpha \in(0,1)$ :

$$
\left\{\begin{array}{l}
\partial_{t} u^{\varepsilon}+u^{\varepsilon} \cdot \nabla u^{\varepsilon}+\varepsilon(-\Delta)^{\frac{\alpha}{2}} u^{\varepsilon}+\nabla P^{\varepsilon}=0, \\
\nabla \cdot u^{\varepsilon}=0, \\
u_{\mid t=0}^{\varepsilon}=u_{0},
\end{array}\right.
$$

where the diffusion term is given by the fractional power of the Laplacian operator. When we neglect the diffusion term, we obtain the Euler equations

$$
\text { (E) }\left\{\begin{array}{l}
\partial_{t} u+u \cdot \nabla u+\nabla P=0, \\
\nabla . u=0, \\
u_{\mid t=0}=u_{0} .
\end{array}\right.
$$

The mathematical study of the Navier-Stokes system was initiated by Leray in his pioneering work [24]. In fact, by using a compactness method, he proved that for any divergence-free initial data $v^{0}$ in the energy space $L^{2}$, there exists a global solution to $\left(N S_{\varepsilon}\right)$. In two dimensions that weak solution was proven to be unique. However, for higher dimensions $(d \geq 3)$ the problem of uniqueness is still widely open. In the 60's, Fujita and Kato [19] constructed for initial data lying in the critical Sobolev space $\dot{H}^{\frac{d}{2}-1}$ a class of unique local solutions called mild solutions. We emphasize that the same result holds true when the initial data belongs to the inhomogeneous Sobolev space $H^{s}$, with $s \geq \frac{d}{2}-1$. The global existence of these solutions is an outstanding open problem. However a positive answer is given at least in both the following cases: either when the initial data is small in the critical space $\dot{H}^{\frac{d}{2}-1}$ which is invariant under the scaling of the Navier-Stokes equations, or in the space dimension two (this is because in two dimensions the scale invariant space is the energy space).

\subsection{The Euler system}

In the two dimensional space and when the regularity is sufficient to give a sense to the Biot-Savart law, then one can consider an alternative weak formulation: the vorticity-stream weak formulation. It consists in resolving the weak form of (1.3) in terms of vorticity $\omega=\operatorname{curl}(u)$ :

$$
\partial_{t} \omega+(u \cdot \nabla) \omega=0,
$$

supplemented with the Biot-Savart law:

$$
u=K * \omega, \quad \text { with } K(x)=\frac{x^{\perp}}{2 \pi|x|^{2}} .
$$

The questions of existence/uniqueness of weak solutions have been extensively studied (see $[10,7,25]$ for instance). We emphasize that, unlike the fixed-point argument, the compactness method does not guarantee the uniqueness of the solutions and then the two issues (existence/uniqueness) are usually dealt with separately. Existence and uniqueness of 
weak solutions to the Euler system were originally addressed by Yudovich in [32] in the context of the Euler equations where the existence and uniqueness of weak solutions to 2D Euler systems (in a bounded domain) are proved under the assumptions: $u_{0} \in L^{2}$ and $\omega_{0} \in L^{\infty}$. Many works have been dedicated to the extension of this result to more general spaces (see [28,15,21,9,30,31,33,14,17,20] for instance). In [6] we have extended Yudovich's result to some class of initial vorticity in a Banach space of BMO-type which is strictly imbricated between $L^{\infty}$ and BMO for which one has the following three fundamental properties: global existence, uniqueness and regularity persistence.

\subsection{The inviscid limit and the main results}

The problem of the convergence of smooth viscous solutions of (1.1) to the Eulerian one as $\varepsilon$ goes to zero is well understood (in the case of the whole space of the torus). Majda showed that under the assumption $v^{0} \in H^{s}$ with $s>d+2$, the solutions $\left(u^{\varepsilon}\right)_{\varepsilon>0}$ converge in $L^{2}$ norm when $\varepsilon$ goes to zero to the unique solution of (1.3). The convergence rate is of order $(\varepsilon t)^{\frac{1}{2}}$. This result has been improved by Masmoudi [26]. For Yudovich type solutions with only the assumption that the vorticity is bounded this question was resolved by Chemin [11].

We now come to describe our results, and we first point out that all the current work is concerned with NavierStokes system in the whole plane $\mathbb{R}^{2}$. The first result of this paper is the following (see Section 2 for the definitions of the spaces).

Theorem 1.1. Assume $p \in[1,2)$. Let $u_{0} \in L^{2}\left(\mathbb{R}^{2}\right)$ be a divergence free vector field such that $\omega_{0} \in L^{p} \cap \mathrm{LBMO}$ and $u_{\varepsilon}($ resp. $u)$ be the solution of $\left(N S_{\varepsilon}\right)($ resp. $(E))$. Then, for every $T>0$ there exist $C=C\left(u_{0}\right)$ and $\varepsilon_{0}=\varepsilon_{0}\left(u_{0}, T\right)$ such that

$$
\left\|u^{\varepsilon}(t)-u(t)\right\|_{L^{2}\left(\mathbb{R}^{2}\right)} \leq(C t \varepsilon)^{\frac{1}{2} \exp \left(1-e^{C t}\right)}, \quad \forall t \in[0, T], \forall \varepsilon \leq \varepsilon_{0}
$$

Remark 1.2. In [6] the global existence and uniqueness for $2 \mathrm{D}$ Euler with initial vorticity $\omega_{0} \in L^{p} \cap$ LBMO have been proved. The additional assumption $u_{0} \in L^{2}\left(\mathbb{R}^{2}\right)$ is easily propagated and we get $u \in L^{\infty} L^{2}$.

The second result is the counterpart version for more regular initial data, with an improved rate of convergence.

Theorem 1.3. Assume $p \in[1,2)$. Let $u_{0} \in L^{2}\left(\mathbb{R}^{2}\right)$ a divergence free vector field such that $\omega_{0}=\operatorname{curl}\left(u_{0}\right) \in L^{p} \cap$ Lmo. Then there exists a unique solution of the $2 D$ incompressible Euler equations (1.3) such that, for every $\delta \in(0,1)$, $\omega \in L_{\mathrm{loc}}^{\infty}\left([0, \infty) ; L^{\alpha} m o \cap L^{p}\right)$, where ${ }^{1}$

$$
\begin{aligned}
\alpha(t) & =1-\sqrt{t}, \quad 0 \leq t \leq \delta^{2}, \\
& =1-\delta, \quad t>\delta^{2} .
\end{aligned}
$$

Moreover for every $T>0$ and every $\delta \in(0,1)$ there exist $C=C\left(u_{0}, \delta\right)$ and $\varepsilon_{0}=\varepsilon_{0}\left(u_{0}, T, \delta\right)$ such that

$$
\left\|u^{\varepsilon}(t)-u(t)\right\|_{L^{2}\left(\mathbb{R}^{2}\right)} \leq(C t \varepsilon)^{\frac{1}{2} \beta(t)}, \quad \forall t \in[0, T], \forall \varepsilon \leq \varepsilon_{0},
$$

with

$$
\beta(t)=\max \left(1-\delta,\left(2-e^{C t}\right)^{\frac{1}{\delta}}\right) .
$$

Remark 1.4. The first part gives the global existence of solutions for the Euler equations, with a loss of regularity as small as we want (since $1-\delta \leq \alpha \leq 1$ and $\delta$ is arbitrary small). This improves some results of [12] in the particular situation of $L^{\alpha} m o$ with $\alpha=1$.

Remark 1.5. The order rates of convergence (of Theorems 1.1 and 1.3) are equal to $\frac{1}{2}$ at $t=0$ and then they are decreasing with the time. Moreover, the order of rate of convergence in Theorem 1.3 is bigger than $\frac{1-\delta}{2}$ (for $\delta$ as small

\footnotetext{
${ }^{1}$ For a given function $\alpha: \mathbb{R} \rightarrow \mathbb{R}^{+}$we say $\omega \in L_{\mathrm{loc}}^{\infty}\left([0, \infty) ; L^{\alpha} m o \cap L^{p}\right)$ if$$
\sup _{t \in[0, T]}\|\omega(t)\|_{L^{\alpha(t)} m o \cap L^{p}}<\infty, \quad \forall T>0
$$ 
as we want) which is just below the optimal rate in the case of $\frac{1}{2}$ strong solutions. This rate beats all of the previous rates of convergence for weak solutions: for example, the rate given in the case of weak solutions with bounded vorticity is exponentially decaying in time [11]. See also [13] and [23].

Remark 1.6. Since the $L^{\infty}$-norm of $\left(u^{\varepsilon}\right)_{\varepsilon>0}$ is uniformly bounded then, by interpolation, the convergence to the Eulerian solution $u$ holds in every $L^{q}$ with $q \in[2,+\infty[$.

The uniform (with respect to the viscosity parameter $\varepsilon$ ) bound of the family of solutions to (1.1) in the adequate space remains essentially open. The difficulty is due to the nature of BMO-type norms which prevents us from dealing with a transport and diffusion at the same time. To overcome this difficulty we use an idea which is based on Trotter's formula: we discretize time and alternate the Euler and Heat equations in small time intervals and then let the length of the interval go to 0 . The implementation of this algorithm is heavily related to the values of the universal constants appearing in the logarithmic estimates. In the favorable case this gives us a local uniform bound of solutions to (1.1) in the adequate space. To explain this let us recall first logarithmic estimates. For $\Phi$ is defined on $] 0,+\infty[\times] 0,+\infty[$ one denotes

$$
\|\psi\|_{K_{\Phi}}:=\sup _{x \neq y} \Phi(|\psi(x)-\psi(y)|,|x-y|),
$$

for every $\psi$ a homeomorphism on $\mathbb{R}^{d}$.

A logarithmic estimate in some functional Banach space $\mathcal{X}$ is of the form

$$
\|f \circ \psi\|_{\mathcal{X}} \leq\left[C_{1}+C_{2} \ln \left(\|\psi\|_{C(\psi)}\right)\right]\|f\|_{\mathcal{X}}
$$

for any Lebesgue measure preserving homeomorphism $\psi$. The constants $C_{1}, C_{2}$ are of course universal and $C(\psi)$ a constant describing the required regularity of $\phi$.

These estimates arise naturally in the study of transport PDEs, associated to a free-divergence vector field. Indeed, such a vector field gives rise to a bi-Lipschitz measure preserving flow, which plays a crucial role for solving the transport equation. In [31] Vishik obtained a logarithmic growth for the Besov space $\left(\mathcal{X}=B_{\infty, 1}^{0}\right.$ and Lipschitzian flow) with applications to Euler equation. More recently, the authors have proven a similar result for $\mathcal{X}=\mathrm{BMO}$ and Lipschitz flows [5] and $\mathcal{X}=L^{p} \cap$ LBMO [6]. In the last case, the flow is not Lipschitz and $\Phi$ is defined by

$$
\Phi(r, s)= \begin{cases}\max \left(\frac{1+|\ln (s)|}{1+|\ln r|}, \frac{1+|\ln r|}{1+|\ln (s)|}\right), & \text { if }(1-s)(1-r) \geq 0, \\ (1+|\ln s|)(1+|\ln r|), & \text { if }(1-s)(1-r) \leq 0 .\end{cases}
$$

In these results the sharp values of $C_{1}$ and $C_{2}$ are not important so no attempt to determine these values were made. Our conjecture about this issue is:

\section{Conjecture 1.7. In both cases considered in [5,6] the constant $C_{1}$ can be taken equal to 1.}

We are able to confirm this conjecture only in the BMO-case and $L^{\alpha} m o$-case with a bi-Lipschitz flow $\phi$. More precisely we have the following improvement of a result in [5] for the composition in BMO.

Theorem 1.8. In $\mathbb{R}^{d}$, there exists a constant $c:=c(d)$ such that for every function $f \in \mathrm{BMO}$ and every measurepreserving bi-Lipschitz homeomorphism $\phi$, we have

$$
\|f \circ \phi\|_{\mathrm{BMO}} \leq\|f\|_{\mathrm{BMO}}\left[1+c \log \left(K_{\phi}\right)\right],
$$

where

$$
K(\phi)=K_{\phi}:=\sup _{x \neq y} \max \left(\frac{|\phi(x)-\phi(y)|}{|x-y|}, \frac{|x-y|}{|\phi(x)-\phi(y)|}\right) .
$$

Remark 1.9. In [5], such result was already obtained with a control by $c_{1}\left[1+c \log \left(K_{\phi}\right)\right]$ with an implicit constant $c_{1}$. The aim here is to improve it by proving that $c_{1}$ may be chosen equal to 1 , which brings an important improvement when the map $\phi$ converges to the identity or any isometry (which is equivalent to $K_{\phi}$ converging to 1 ). 
As an application, our second result is then the following:

Theorem 1.10. Take $p \in[1,2)$ and $\alpha>1$ and set $\mathcal{B}_{p, \alpha}:=L^{p} \cap L^{\alpha}$ mo. Then for every $u_{0} \in L^{2}\left(\mathbb{R}^{2}\right)$ a divergence free vector field such that $\omega_{0}=\operatorname{rot} u_{0} \in \mathcal{B}_{p, \alpha}$ there exist $T=T\left(\left\|\omega_{0}\right\|_{\mathcal{B}_{p, \alpha}}\right)$ and $C_{0}=C_{0}\left(\left\|\omega_{0}\right\|_{\mathcal{B}_{p, \alpha}}\right)$ such that the family $\left(u^{\varepsilon}\right)_{\varepsilon>0}$ of solutions to (1.1) satisfies the following bounds uniformly with respect to $\varepsilon>0$ :

$$
\left\|u^{\varepsilon}\right\|_{L^{\infty}\left([0, T], L^{2}\right)}+\left\|\operatorname{rot}\left(u^{\varepsilon}\right)\right\|_{L^{\infty}\left([0, T], \mathcal{B}_{p, \alpha}\right)} \leq C_{0} .
$$

The same holds for the fractional Navier-Stokes equations (1.2).

The remainder of this paper is organized as follows. In the next section we describe some preliminaries about functional spaces and how they appear in the study of 2D Euler equation. Theorem 1.1 is proved in Section 3. Section 4 is devoted to the study of the Euler equations with initial vorticity in Lmo, Theorem 1.3. Then in Section 5, we prove Theorem 1.10 by a discretization scheme.

\section{Definitions and preliminaries on functional spaces}

In this preparatory section we recall some definitions of useful functional spaces and we give some results which are needed later.

\subsection{The scale of $L^{\alpha}$ mo spaces}

We first define the $L^{\alpha}$ mo spaces.

Definition 2.1. Let $\alpha \in[0, \infty)$ and $f: \mathbb{R}^{2} \rightarrow \mathbb{R}$ be a locally integrable function. We say that $f$ belongs to $L^{\alpha} m o$ if

$$
\|f\|_{L^{\alpha} m o}:=\sup _{0<r \leq \frac{1}{2}}|\ln r|^{\alpha}\left(\operatorname{Avg}_{B}\left|f-\operatorname{Avg}_{B} f\right|^{2}\right)^{\frac{1}{2}}+\left(\sup _{|B|=1} \int_{B}|f(x)|^{2} d x\right)^{\frac{1}{2}}<\infty,
$$

where the first supremum is taken over all the balls $B$ of radius $r \leq \frac{1}{2}$. For convenience, $L^{1}$ mo is denoted by $L m o$.

Remark 2.2. As dictated by a variant of John-Nirenberg inequalities (see [18,3]), if we replace the $L^{2}$-control of the oscillations by an $L^{p}$-control for some $p \in[1,2)$ then we obtain an equivalent norm.

We also recall the functional space LBMO, introduced in [6].

Definition 2.3. The LBMO-norm is defined by

$$
\|f\|_{\mathrm{LBMO}}:=\|f\|_{\mathrm{BMO}}+\sup _{B_{1}, B_{2}} \frac{\left|\operatorname{Avg}_{B_{2}}(f)-\operatorname{Avg}_{B_{1}}(f)\right|}{1+\ln \left(\frac{1-\ln r_{B_{2}}}{1-\ln r_{B_{1}}}\right)},
$$

where the supremum is taken over all pairs of balls $B_{1}$ and $B_{2}$ in $\mathbb{R}^{2}$ with $0<r_{B_{1}} \leq 1$ and $2 B_{2} \subset B_{1}$.

Remark 2.4. We gather here some easy properties of these spaces:

(i) All the spaces defined above are Banach spaces.

(ii) If $\alpha<\beta$ then $L^{\beta} m o \subset L^{\alpha} m o$.

(iii) If $\alpha=0$ then $L^{\alpha} m o$ corresponds to the intersection between bmo (the local BMO space) and $L_{u l o c}^{1}$ (the space of uniformly locally integrable functions).

(iv) For $\alpha \geq 0$ and $p \in(1, \infty)$, the space $L^{\alpha} m o \cap L^{p}$ is included in $\mathrm{BMO} \cap L^{p}$.

(v) The convolution operator by an $L^{1}$-normalized function is a contraction on all these spaces.

The following lemma will be used in the sequel. 
Lemma 2.5. For $\alpha>1$, we have the continuous embedding $L^{\alpha}$ mo $\hookrightarrow L^{\infty}$. The condition $\alpha>1$ is optimal, since there exist non-bounded functions belonging to Lmo.

Proof. Let $x$ be a fixed point of $\mathbb{R}^{2}$ and consider $B(r)=B(x, r)$ the balls centered at $x$. Then for a function $f \in L^{\alpha} m o$, it is well-known that we have for $n \gg 1$

$$
\begin{aligned}
\left|\operatorname{Avg}_{B\left(2^{-n}\right)} f-\operatorname{Avg}_{B(1)} f\right| & \leq \sum_{k=1}^{n}\left|\operatorname{Avg}_{B\left(2^{-k}\right)} f-\operatorname{Avg}_{B\left(2^{-k+1}\right)} f\right| \\
& \leq\|f\|_{L^{\alpha} m o} \sum_{k=1}^{n}(1+k)^{-\alpha} .
\end{aligned}
$$

Since $\alpha>1$ then the sum is convergent and so we deduce that

$$
\limsup _{n \rightarrow \infty}\left|\operatorname{Avg}_{B\left(2^{-n}\right)} f\right| \lesssim\|f\|_{L^{\alpha} m o} .
$$

Since $f$ is locally integrable, the differentiation theorem allows us to conclude that $f \in L^{\infty}$ and

$$
\|f\|_{L^{\infty}} \lesssim\|f\|_{L^{\alpha} m o} .
$$

For the sharpness of the result, we refer to [4, Proposition 2] where the function $x \mapsto \log (1-\log (|x|)) \mathbf{1}_{|x| \leq 1}$ is shown to belong to Lmo, in $\mathbb{R}^{2}$.

We also need the following result:

Proposition 2.6. For every $\alpha \geq 0$ and $p \in(1, \infty)$, the space $L^{\alpha}$ mo $\cap L^{p}$ is stable by the action of any Riesz transforms.

We do not write the proof since it is essentially the same as the one in [27] (Theorem 1.1) except that we work here with the local version of BMO-type spaces. What happens on the larger balls (ball of radius larger than 1) can be easily studied using the $L^{p}$ norm.

In the sequel we will use the following interpolation lemma. ${ }^{2}$

Lemma 2.7. There exists $C=C(n)$ such that the following estimate holds for every $r \in[2,+\infty)$ and every smooth function $f$

$$
\|f\|_{L^{r}} \leq C r\|f\|_{L^{2} \cap \mathrm{BMO}} .
$$

Proof. We consider the usual Hardy-Littlewood maximal operator:

$$
M(f)(x)=\sup _{B \ni x} \frac{1}{|B|} \int_{B}|f(x)| d x=\sup _{B \ni x} \operatorname{Avg}_{B}|f| .
$$

Let $\lambda:=\|f\|_{\mathrm{BMO}_{r}}$, where $\mathrm{BMO}_{r}$ is the $\mathrm{BMO}$-norm with oscillations controlled in $L^{r}$ and set

$$
E_{\lambda}=\{x: M(f)(x)>\lambda\} .
$$

Let $\left(Q_{i}\right)_{i}$ be a Whitney covering of $E_{\lambda}$. We have in particular

$$
Q_{i} \subset E_{\lambda} \text { and } 4 Q_{i} \cap E_{\lambda}^{c} \neq \varnothing,
$$

which implies

$$
\operatorname{Avg}_{4 Q_{i}}|f| \leq \lambda \quad \text { and so } \quad \operatorname{Avg}_{Q_{i}}(f) \leq 4^{n} \lambda
$$

\footnotetext{
2 The main point in this lemma is the linear dependence of the interpolation constant. Actually, the interpolation itself is well known [22] but we haven't found in the literature this type of constants. For the sake of completeness we give the proof.
} 
One has, for every $i \in \mathbb{N}$,

$$
\begin{aligned}
\left(\int_{Q_{i}}|f(x)|^{r} d x\right)^{1 / r} & \leq\left(\int_{Q_{i}}\left|f(x)-\operatorname{Avg}_{Q_{i}}(f)\right|^{r} d x\right)^{1 / r}+4^{n} \lambda\left|Q_{i}\right|^{1 / r} \\
& \leq\left|Q_{i}\right|^{1 / r}\left(\|f\|_{\mathrm{BMO}_{r}}+4^{n} \lambda\right) \\
& \leq C_{n}\left|Q_{i}\right|^{1 / r}\|f\|_{\mathrm{BMO}_{r}},
\end{aligned}
$$

where $C=C_{n}$ is a constant depending only on the dimension $n$. Summing on $i \in \mathbb{N}$ one gets

$$
\begin{aligned}
\int_{E_{\lambda}}|f(x)|^{r} d x & \leq \sum_{i} \int_{Q_{i}}|f(x)|^{r} d x \\
& \lesssim C_{n}^{r}\left|E_{\lambda}\right|\|f\|_{\mathrm{BMO}_{r}}^{r},
\end{aligned}
$$

where $C$ is a universal constant. But, by maximal theorem (see [22] for instance),

$$
\left|E_{\lambda}\right| \lesssim \lambda^{-2}\|f\|_{L^{2}}^{2}
$$

This gives

$$
\begin{aligned}
\int_{E_{\lambda}}|f(x)|^{r} d x & \leq C_{n}^{r} \lambda^{-2}\|f\|_{L^{2}}\|f\|_{\mathrm{BMO}_{r}}^{r} \\
& \leq C_{n}^{r}\|f\|_{L^{2}}\|f\|_{\mathrm{BMO}_{r}}^{r-2} .
\end{aligned}
$$

Trivially one has

$$
|f(x)| \leq M(f)(x) \leq \lambda, \quad \forall x \in E_{\lambda}^{c} .
$$

This yields, via Hölder inequality,

$$
\begin{aligned}
\int_{E_{\lambda}^{C}}|f(x)|^{r} d x & \leq\left(\int_{E_{\lambda}^{C}}|f(x)|^{2} d x\right)\|f\|_{L^{\infty}\left(E_{\lambda}^{c}\right)}^{r-2} \\
& \leq\|f\|_{L^{2}}^{2} \lambda^{r-2} \\
& \leq\|f\|_{L^{2}}^{2}\|f\|_{\mathrm{BMO}_{r}}^{r-2} .
\end{aligned}
$$

Finally, we get

$$
\int|f(x)|^{r} d x \leq C^{r}\|f\|_{L^{2}}\|f\|_{\mathrm{BMO}_{r}}^{r-2}
$$

where $C=C(n)$ is a universal constant.

One of the direct consequences of the John-Nirenberg inequality is (with $\Gamma$-function satisfying $\Gamma(r) \lesssim r^{r}$ )

$$
\begin{aligned}
\|f\|_{\mathrm{BMO}_{r}\left(\mathbb{R}^{n}\right)} & \lesssim_{n}(r \Gamma(r))^{\frac{1}{r}}\|f\|_{\mathrm{BMO}\left(\mathbb{R}^{n}\right)} \\
& \lesssim_{n} r\|f\|_{\mathrm{BMO}\left(\mathbb{R}^{n}\right) .}
\end{aligned}
$$

Thus, we obtain finally

$$
\begin{aligned}
\|f\|_{L^{r}} & \lesssim n r^{1-\frac{2}{r}}\|f\|_{L^{2}}^{\frac{2}{r}}\|f\|_{\mathrm{BMO}}^{1-\frac{2}{r}} \\
& \lesssim n r\|f\|_{L^{2}}^{\frac{2}{r}}\|f\|_{\mathrm{BMO}}^{1-\frac{2}{r}},
\end{aligned}
$$

as claimed. 


\subsection{Regularity estimates on the flow for $L^{\alpha}$ mo vorticity}

We first aim to obtain information on the regularity of the velocity vector-field $u$, associated to an $L^{\alpha} m o$-vorticity $\omega$ via the Biot-Savart law:

$$
u=K * \omega, \quad \text { with } K(x)=\frac{x^{\perp}}{2 \pi|x|^{2}} .
$$

Let us first recall the following definition.

Definition 2.8. We say that a function $f: \mathbb{R}^{2} \rightarrow \mathbb{R}^{2}$ belongs to the class $L^{\beta} L$ for $\beta \in[0,1]$ if

$$
\|f\|_{L^{\beta} L}:=\sup _{0<|x-y|<\frac{1}{2}} \frac{|f(x)-f(y)|}{|x-y||\ln | x-\left.y\right|^{\beta}}+\|f\|_{L^{\infty}}<\infty .
$$

Note also that the space $L^{\beta} L$ may also be equipped with the following equivalent norm:

$$
\|f\|_{L^{\beta} L} \simeq \sup _{x \neq y} \frac{|f(x)-f(y)|}{|x-y|\left(1+|\ln | x-\left.y\right|^{\beta}\right)}+\|f\|_{L^{\infty}} .
$$

For $\beta=0$, this corresponds to bounded and Lipschitz functions and so $L^{0} L$ will be denoted by Lip.

We first give a refinement of [4, Proposition 5].

Proposition 2.9. For $p \in(1,2)$, there exists a constant $\rho$ such that for every $\alpha \geq 0$, and every vorticity $\omega \in L^{\alpha} m o \cap L^{p}$ the corresponding velocity $u$ given by (2.1) satisfies the following:

- If $\alpha \in[0,1)$ then $u \in L^{1-\alpha} L$ with

$$
\|u\|_{L^{1-\alpha} L} \leq \frac{\rho}{1-\alpha}\|\omega\|_{L^{\alpha} m o \cap L^{p}}
$$

- If $\alpha>1$ then $u \in$ Lip with

$$
\|u\|_{\text {Lip }} \leq \frac{\rho \alpha}{\alpha-1}\|\omega\|_{L^{\alpha} m o \cap L^{p}} .
$$

Proof. The $L^{\infty}$-norm of $u$ can be easily bounded. Actually, a direct consequence of the Biot-Savart law yields

$$
\begin{aligned}
\|u\|_{L^{\infty}} & \leq\left\|K \mathbb{1}_{|x|<1}\right\|_{L^{p}}\|\omega(t)\|_{L^{p^{\prime}}}+\left\|K \mathbb{1}_{|x| \geq 1}\right\|_{L^{p^{\prime}}}\|\omega(t)\|_{L^{p}} \\
& \leq C_{p}\left(\|\omega(t)\|_{L^{\alpha} m o}+\|\omega(t)\|_{L^{p}}\right),
\end{aligned}
$$

where $p^{\prime}$ is the conjugate exponent of $p$ and where we used (since $1<p<2$ ) that $L^{p^{\prime}} \subset L^{p} \cap \mathrm{BMO} \subset L^{p} \cap L^{\alpha} m o$.

For $\alpha \in[0,1)$, we follow the same proof as in [4, Propositions 1 and 5] and keep track of the behavior of implicit constants with respect to $\alpha$ (more precisely, we use that $\sum_{n=1}^{N} n^{-\alpha} \lesssim \frac{1}{1-\alpha} N^{1-\alpha}$ ).

For $\alpha>1$, consider $\omega \in L^{\alpha} m o$. Then denote by $S_{0},\left(\Delta_{n}\right)_{n \geq 0}$ the standard Littlewood-Paley projectors. The following inequality holds (see [4]):

$$
\left\|\Delta_{n} \omega\right\|_{L^{\infty}} \lesssim(1+n)^{-\alpha}\|\omega\|_{L^{\alpha} m o} .
$$

Invoking Bernstein inequality and the well-known ${ }^{3}\left\|\nabla \Delta_{n} u\right\|_{L^{\infty}} \simeq\left\|\Delta_{n} \omega\right\|_{L^{\infty}}$

$$
|u(x)-u(y)| \leq|x-y|\left\|\nabla S_{0}(u)\right\|_{L^{\infty}}+|x-y| \sum_{n \geq 0}\left\|\nabla \Delta_{n} u\right\|_{L^{\infty}}
$$

$\overline{3}$ We recall that $\nabla u=\mathcal{R}(\omega)$ for some Riesz transform $\mathcal{R}$. 


$$
\begin{aligned}
& \leq|x-y|\|u\|_{L^{\infty}}+|x-y| \sum_{n \geq 0}\left\|\Delta_{n} \omega\right\|_{L^{\infty}} \\
& \leq|x-y|\left(\|u\|_{L^{\infty}}+\|\omega\|_{L^{\alpha} m o} \sum_{n \geq 0}(1+n)^{-\alpha}\right) .
\end{aligned}
$$

The first part of the proof and the easy fact $\sum_{n \geq 0}(1+n)^{-\alpha} \simeq \frac{\alpha}{\alpha-1}$ conclude the proof.

For a time-dependent divergence-free vector-field $u:=\mathbb{R}^{+} \times \mathbb{R}^{2} \rightarrow \mathbb{R}^{2}$ we define the flow $\psi(t, \cdot)$ as the solution of the differential equation

$$
\partial_{t} \psi(t, x)=u(t, \psi(t, x)), \quad \psi(0, x)=x .
$$

We have the following regularity:

Proposition 2.10. (See [4, Proposition 6].) Let u be a smooth divergence-free vector field and $\psi$ be its flow (and $\psi^{-1}$ its inverse). Then there exists a constant $\eta$ (independent of $u$ ) such that for every non-increasing function $\alpha: \mathbb{R}^{+} \rightarrow$ $(0,1]$ and for every $t \geq 0$ we have

$$
|x-y| \neq 0 \Longrightarrow\left|\psi^{ \pm 1}(t, x)-\psi^{ \pm 1}(t, y)\right| \leq|x-y| e^{\eta V(t)|\ln | x-y||^{1-\alpha(t)}},
$$

where

$$
V(t):=\int_{0}^{t}\|u(\tau)\|_{L^{1-\alpha(\tau)} L} d \tau
$$

We do not repeat the proof, since it is exactly the same one as detailed in [4, Proposition 6], where the implicit constants are shown to be independent of $\alpha$.

Similarly we have the same for a Lipschitz-velocity, which is more well-known:

Proposition 2.11. Let $u$ be a smooth divergence-free vector field and $\psi$ be its flow (and $\psi^{-1}$ its inverse). Then there exists a constant $\eta$ (independent of $u$ ) such that for every $t \geq 0$ we have

$$
\left\|\psi^{ \pm 1}(t, \cdot)\right\|_{\text {Lip }} \leq e^{V(t)}
$$

where

$$
V(t):=\int_{0}^{t}\|u(\tau)\|_{\text {Lip }} d \tau
$$

\section{Inviscid limit for an initial vorticity in LBMO, Theorem 1.1}

Proof of Theorem 1.1. This proof, which follows a rather classical scheme, ${ }^{4}$ is based on two main ingredients: the control of the BMO-norm of the solution of $(E)$ (proved in [6]) and the refined expression of the constant appearing in Lemma 2.7.

It is well-known since [24] that the bidimensional Navier-Stokes system (1.1) with initial velocity in $L^{2}$ has a unique solution $u^{\varepsilon}$ satisfying

$$
\left\|u^{\varepsilon}(t)\right\|_{L^{2}}^{2}+2 \varepsilon \int_{0}^{t}\left\|\nabla u^{\varepsilon}\left(t^{\prime}\right)\right\|_{L^{2}}^{2} d t^{\prime}=\left\|u_{0}\right\|_{L^{2}}^{2}, \quad \forall t \geq 0 .
$$

\footnotetext{
4 See [11] for instance.
} 
The vorticity $\omega^{\varepsilon}:=\partial_{1} u_{2}^{\varepsilon}-\partial_{2} u_{1}^{\varepsilon}$ satisfies the following convection-diffusion equation

$$
\partial_{t} \omega^{\varepsilon}+u^{\varepsilon} \cdot \nabla \omega^{\varepsilon}-\varepsilon \Delta \omega^{\varepsilon}=0, \quad \omega_{\mid t=0}^{\varepsilon}=\omega_{0} .
$$

The classical $L^{p}$ estimate for this equation yields

$$
\left\|\omega^{\varepsilon}(t)\right\|_{L^{p}} \leq\left\|\omega_{0}\right\|_{L^{p}}, \quad \forall t \geq 0 .
$$

Let $U^{\varepsilon}=u^{\varepsilon}-u$ and $\pi^{\varepsilon}=P^{\varepsilon}-P$. One denotes also $\Omega^{\varepsilon}=\omega^{\varepsilon}-\omega$, where $\omega^{\varepsilon}$ is the vorticity of $u^{\varepsilon}$ and $\omega$ is the vorticity of $u$.

The vector field $U^{\varepsilon}$ satisfies

$$
\left\{\begin{array}{l}
\partial_{t} U^{\varepsilon}+u^{\varepsilon} \cdot \nabla U^{\varepsilon}+\nabla \pi^{\varepsilon}=U^{\varepsilon} \cdot \nabla u+\varepsilon \Delta u^{\varepsilon}, \quad x \in \mathbb{R}^{2}, t>0, \\
\nabla . U^{\varepsilon}=0, \\
U_{\mid t=0}^{\varepsilon}=0 .
\end{array}\right.
$$

The energy estimate gives

$$
\begin{aligned}
\frac{d}{d t}\left\|U^{\varepsilon}\right\|_{L^{2}}^{2} & \leq\left|\left\langle U^{\varepsilon} \cdot \nabla u, U^{\varepsilon}\right\rangle\right|+\varepsilon\left\|\nabla u^{\varepsilon}\right\|_{L^{2}}\left\|\nabla U^{\varepsilon}\right\|_{L^{2}} \\
& \leq I+I I .
\end{aligned}
$$

By $L^{2}$-continuity of the Riesz-operator one has, for every $t \in[0, T]$,

$$
\begin{aligned}
I I & \leq \varepsilon\left\|\omega^{\varepsilon}\right\|_{L^{2}}\left(\left\|\omega^{\varepsilon}\right\|_{L^{2}}+\|\omega\|_{L^{2}}\right) \\
& \leq \varepsilon C_{0} .
\end{aligned}
$$

The last estimate follows from the uniform bound of the $L^{2}$ norm of the vorticities. ${ }^{5}$

On the other hand, by Hölder inequality and the continuity of the Riesz-operator one gets, for every $q \geq 2$,

$$
\begin{aligned}
I & \leq \int_{\mathbb{R}^{2}}\left|\nabla u(t, x) \| U^{\varepsilon}(t, x)\right|^{2} d x \\
& \leq\|\nabla u\|_{L^{q}}\left\|U^{\varepsilon}\right\|_{L^{2 q^{\prime}}}^{2} .
\end{aligned}
$$

Using Lemma 2.7 we infer

$$
I \lesssim q\|\nabla u\|_{L^{2} \cap \mathrm{BMO}}\left\|U^{\varepsilon}\right\|_{L^{2 q^{\prime}}}^{2}
$$

The continuity of the Riesz operator on $L^{2} \cap \mathrm{BMO}$ yields ${ }^{6}$

$$
\begin{aligned}
I & \lesssim q\|\omega(t)\|_{L^{2} \cap \mathrm{BMO}}\left\|U^{\varepsilon}(t)\right\|_{L^{2 q^{\prime}}}^{2} \\
& \leq q C_{0} e^{C_{0} t}\left\|U^{\varepsilon}(t)\right\|_{L^{2 q^{\prime}}}^{2},
\end{aligned}
$$

where we have used Theorem 1.1 in [6] $\left(C_{0}=C_{0}\left(\left\|\omega_{0}\right\|_{L^{p} \cap L B M O}\right)\right)$. Using the Hölder inequality and the Biot-Savart law one obtains

$$
\begin{aligned}
\left\|U^{\varepsilon}\right\|_{L^{2 q^{\prime}}}^{2} & \lesssim\left\|U^{\varepsilon}\right\|_{L^{\infty}}^{\frac{2}{q}}\left\|U^{\varepsilon}\right\|_{L^{2}}^{2-\frac{2}{q}} \\
& \lesssim\left\|\Omega^{\varepsilon}\right\|_{L^{p} \cap L^{3}}^{\frac{2}{q}}\left\|U^{\varepsilon}\right\|_{L^{2}}^{2-\frac{2}{q}} \\
& \lesssim\left(\left\|\omega^{\varepsilon}\right\|_{L^{p} \cap L^{3}}+\|\omega\|_{L^{p} \cap L^{3}}\right)^{\frac{2}{q}}\left\|U^{\varepsilon}\right\|_{L^{2}}^{2-\frac{2}{q}} .
\end{aligned}
$$

Since $\left\|\omega^{\varepsilon}(t)\right\|_{L^{p} \cap L^{3}}^{\frac{2}{q}}$ is uniformly bounded then the outcome is

\footnotetext{
5 By interpolation between $L^{p}$ and BMO we know that $\omega_{0} \in L^{r}$ for every $r \in[p,+\infty[$.

6 The continuity of a Riesz operator on BMO was proved in [27], see also Proposition 2.6.
} 


$$
\frac{d}{d t}\left\|U^{\varepsilon}(t)\right\|_{L^{2}}^{2} \leq C_{0}\left(\varepsilon+q e^{C_{0} t}\left\|U^{\varepsilon}\right\|_{L^{2}}^{2-\frac{2}{q}}\right),
$$

for all $q \geq 2$ and some constant $C_{0}=C_{0}\left(\left\|\omega_{0}\right\|_{L^{p} \cap \mathrm{LBMO}}\right)$.

Take $g^{\varepsilon}(t):=\left\|U^{\varepsilon}(t)\right\|_{L^{2}}^{2}$ and define $T^{\varepsilon}<T$ the maximal time:

$$
T^{\varepsilon}:=\max \left\{t \leq T: \sup _{\tau \in[0, t]} g^{\varepsilon}(\tau) \leq \frac{1}{e^{2}}\right\} .
$$

For every $t \in\left(0, T^{\varepsilon}\right)$ one chooses $q=-\ln \left(g^{\varepsilon}(t)\right)$ in (3.1) to get

$$
\dot{g}^{\varepsilon}(t) \leq C_{0}\left(\varepsilon-e^{C_{0} t} \ln \left(g^{\varepsilon}(t)\right) g^{\varepsilon}(t)\right) .
$$

Integrating this differential inequality, we get

$$
g^{\varepsilon}(t) \leq C_{0} \varepsilon t+\int_{0}^{t}-C_{0} e^{C_{0} t^{\prime}} \ln \left(g^{\varepsilon}\left(t^{\prime}\right)\right) g^{\varepsilon}\left(t^{\prime}\right) d t^{\prime} .
$$

Assuming $C_{0} T \varepsilon_{0}<1$ and applying Lemma 3.1 below, we get

$$
-\ln \left(-\ln \left(g^{\varepsilon}\right)\right)+\ln \left(-\ln \left(C_{0} t \varepsilon\right)\right) \leq\left(e^{C_{0} t}-1\right), \quad \forall t<T^{\varepsilon} .
$$

This yields, for all $t<T^{\varepsilon}$

$$
g^{\varepsilon}(t) \leq\left(C_{0} t \varepsilon\right)^{\beta(t)},
$$

with $\beta(t)=\exp \left(1-e^{C_{0} t}\right)$. In particular,

$$
g^{\varepsilon}(t) \leq\left(C_{0} t \varepsilon\right)^{\beta(t)}, \quad \forall t \in\left[0, T^{\varepsilon}[.\right.
$$

If we assume that $\varepsilon_{0}$ satisfies also

$$
\left(C_{0} T \varepsilon_{0}\right)^{\beta(T)} \leq \frac{1}{e^{2}}
$$

we get $T^{\varepsilon}=T$, for all $\varepsilon \leq \varepsilon_{0}$.

This gives finally and so

$$
g^{\varepsilon}(t) \leq\left(C_{0} t \varepsilon\right)^{\beta(t)}, \quad \forall t \in[0, T], \varepsilon \leq \varepsilon_{0},
$$

for some constant $C_{0}=C_{0}\left(\left\|\omega_{0}\right\|_{L^{p} \cap \mathrm{LBMO}}\right)$ and $\varepsilon_{0}=\varepsilon_{0}\left(\left\|\omega_{0}\right\|_{L^{p} \cap \mathrm{LBMO}}, T\right)$, as claimed.

The following Osgood lemma is a slight generalization of [2, Lemma 3.4] for which the function $c$ is constant and its proof is an easy application of it.

Lemma 3.1 (Osgood lemma). Let $\rho$ be a measurable function from $\left[t_{0}, T\right]$ to $[0, a], \gamma$ a locally integrable function from $\left[t_{0}, T\right]$ to $\mathbb{R}^{+}$, and $\mu$ a continuous and nondecreasing function from $[0, a]$ to $\mathbb{R}^{+}$. Assume that, for some nonnegative nondecreasing continuous $c$, the function $\rho$ satisfies

$$
\rho(t) \leq c(t)+\int_{t_{0}}^{t} \gamma\left(t^{\prime}\right) \mu\left(\rho\left(t^{\prime}\right)\right) d t^{\prime} .
$$

Then

$$
-\mathcal{M}(\rho(t))+\mathcal{M}(c(t)) \leq \int_{t_{0}}^{t} \gamma\left(t^{\prime}\right) d t^{\prime},
$$

with

$$
\mathcal{M}(x)=\int_{x}^{a} \frac{1}{\mu(r)} d r .
$$




\section{Inviscid limit for an initial vorticity in Lmo}

\subsection{Existence and uniqueness of global solution for Euler equation with an initial vorticity in Lmo}

In this section we will use Propositions 2.9 and 2.10 to prove that if we solve the 2D Euler equations with initial vorticity $\omega_{0} \in L m o=L^{1} m o$ then given $\delta>0, \omega(t) \in L^{\alpha(t)} m o$ where $\alpha(t)$ is a continuous function with $\alpha(0)=1$ and $\alpha(t) \geq 1-\delta$ for all $0<t<\infty$. Prior to stating the precise theorem, we will make a few comments on the previous results in this direction. It was proven in Vishik [31] that if $\omega_{0}$ satisfies

$$
\sum_{-1}^{n}\left\|\Delta_{j} \omega_{0}\right\|_{L^{\infty}} \lesssim \Pi(n),
$$

with a positive and increasing function $\Pi$ which is such that the series $\sum_{n \geq 2} \Pi(n)^{-1}$ diverges, then we can solve the Euler equations globally in time and for every $t>0$ we have

$$
\sum_{-1}^{n}\left\|\Delta_{j} \omega(t)\right\|_{L^{\infty}} \lesssim n \Pi(n),
$$

where the constant gets worse in time depending upon $\omega_{0}$.

In particular, this result in Besov spaces flavor proves some propagation of the initial regularity but with a loss.

Let us then consider the space Lmo. It is easy to see that for $\omega_{0} \in L m o$ then (4.1) is satisfied with $\Pi(n)=\log (n)$. Applying Vishik's result gives us a solution of Euler equations satisfying (4.2). We claim that indeed the solution is better and satisfies for $t>0$ and any $\delta>0$

$$
\sum_{-1}^{n}\left\|\Delta_{j} \omega(t)\right\|_{L^{\infty}} \lesssim n^{\delta}
$$

(still with implicit constants depending on time and on $\omega_{0}$ ). This will be a consequence of the following theorem (since $\omega(t) \in L^{1-\delta} m o$ implies (4.3)). So by this way, Lmo appears as a subspace of vorticities satisfying (4.1) with $\Pi=\log$ where we improve Vishik's result and the loss of regularity is as small as we want (in terms of exponent of $n$ in (4.3), improving (4.2)).

We now state the main theorem of this section.

Theorem 4.1. Let $0<\delta<1$ and $p \in[1,2)$ be given. Suppose that $\omega_{0} \in$ Lmo $\cap L^{p}$. Then there exists a unique solution of the $2 D$ incompressible Euler equations such that $\omega \in L_{\mathrm{loc}}^{\infty}\left([0, \infty) ; L^{\alpha}\right.$ mo $\left.\cap L^{p}\right)$, where

$$
\begin{aligned}
\alpha(t) & =1-\sqrt{t}, \quad 0 \leq t \leq \delta^{2}, \\
& =1-\delta, \quad t>\delta^{2} .
\end{aligned}
$$

Moreover, for some constant $C=C\left(\omega_{0}\right)$, we have

$$
\|\omega(t)\|_{L^{\alpha(t)} m o \cap L^{p}} \leq C_{0} \exp \left(\frac{C_{0} t}{\delta}\right) .
$$

Remark 4.2. We can take any function $\alpha$ of the form

$$
\begin{aligned}
\alpha(t) & =1-t^{\rho}, \quad 0 \leq t \leq \delta^{1 / \rho}, \\
& =1-\delta, \quad t>\delta^{1 / \rho},
\end{aligned}
$$

for some $\rho \in(0,1)$ and $\delta>0$.

The reason that $\rho=1$ is not admissible is that the regularity loss must be enough so that $\frac{1}{1-\alpha(t)}$ is integrable near $t=0$, as will be clear from the proof. We do not believe that this is an artifact of our proof.

The proof of Theorem 4.1 relies on Proposition 2.9 and Proposition 2.10 as well as the following:

Proposition 4.3. Let $p \in[1,2), \alpha \in(0,1)$ and $\psi$ be a homeomorphism (preserving the measure) such that for every $x \neq y$ 


$$
\left|\psi^{ \pm 1}(x)-\psi^{ \pm 1}(y)\right| \leq|x-y| e^{V|\ln | x-y||^{1-\alpha}},
$$

for some constant $V$. Then for every $\omega_{0} \in L^{\alpha}$ mo $\cap L^{p}$

$$
\left\|\omega_{0}\left(\psi^{-1}\right)\right\|_{L^{\alpha} m o \cap L^{p}} \lesssim(1+V)\left\|\omega_{0}\right\|_{L^{\alpha} m o \cap L^{p} .}
$$

We then easily deduce the following corollary:

Corollary 4.4. Let fix $p \in[1,2), \alpha \in(0,1)$ and $\psi$ be a homeomorphism (preserving the measure) such that for every $x \neq y$

$$
\left|\psi^{ \pm 1}(x)-\psi^{ \pm 1}(y)\right| \leq|x-y| e^{V|\ln | x-y||^{1-\alpha}}
$$

for some constants $V$, $\alpha$. Then for every $\omega_{0} \in \operatorname{Lmo} \cap L^{p}$,

$$
\left\|\omega_{0}\left(\psi^{-1}\right)\right\|_{L^{\alpha} m o \cap L^{p}} \lesssim(1+V)\left\|\omega_{0}\right\|_{L m o \cap L^{p}}
$$

We only prove Proposition 4.3.

Proof. Since $\psi$ preserves the measure, the $L^{p}$ norm is conserved. Hence, we only have to deal with the homogeneous part of the $L^{\alpha} m o$-norm. Let $B$ be a ball of radius $r \leq \frac{1}{2}$ then

$$
\begin{aligned}
\operatorname{Avg}_{B}\left|f-\operatorname{Avg}_{B} f\right| & \leq\left(\operatorname{Avg}_{B}\left|f-\operatorname{Avg}_{B} f\right|^{2}\right)^{\frac{1}{2}} \\
& \leq \inf _{C}\left(\operatorname{Avg}_{B}|f-C|^{2}\right)^{\frac{1}{2}},
\end{aligned}
$$

where the infimum is taken over all the constants $C>0$.

Applying this inequality for $f=\omega=\omega_{0}\left(\psi^{-1}\right)$, it comes for every $q \geq 2$ and every $C>0$,

$$
\begin{aligned}
\operatorname{Avg}_{B}\left|\omega-\operatorname{Avg}_{B} \omega\right| & \leq\left(\operatorname{Avg}_{B}\left|\omega_{0}(\psi)-C\right|^{q}\right)^{\frac{1}{q}} \\
& \leq\left(\operatorname{Avg}_{\psi(B)}\left|\omega_{0}-C\right|^{q}\right)^{\frac{1}{q}} .
\end{aligned}
$$

Due to the modulus regularity of $\psi$, if $B$ is a ball of radius $r$ then $\psi(B)$ is included in $\tilde{B}$ a ball of radius

$$
\tilde{r}:=r e^{V|\ln r|^{1-\alpha}} .
$$

So, for every $C>0$

$$
\begin{aligned}
\operatorname{Avg}_{B}\left|\omega-\operatorname{Avg}_{B} \omega\right| & \leq\left(\frac{|\tilde{B}|}{|B|}\right)^{\frac{1}{q}}\left(\operatorname{Avg}_{\tilde{B}}\left|\omega_{0}-C\right|^{p}\right)^{\frac{1}{q}} \\
& \leq\left(\frac{\tilde{r}}{r}\right)^{\frac{2}{q}}\left(\operatorname{Avg}_{\tilde{B}}\left|\omega_{0}-C\right|^{q}\right)^{\frac{1}{q}} \\
& \leq e^{\frac{2}{q} V|\ln r|^{1-\alpha}}\left(\operatorname{Avg}_{\tilde{B}}\left|\omega_{0}-C\right|^{q}\right)^{\frac{1}{q}} .
\end{aligned}
$$

Then we may chose $C=\operatorname{Avg}_{\tilde{B}} \omega_{0}$ and using the $L^{\alpha} m o_{q}$ regularity of $\omega_{0}$, we obtain

$$
\operatorname{Avg}_{B}\left|\omega-\operatorname{Avg}_{B} \omega\right| \leq e^{\frac{2}{q} V|\ln r|^{1-\alpha}}|\ln r|^{-\alpha}\left\|\omega_{0}\right\|_{L^{\alpha} m o_{q}},
$$

where $L^{\alpha} m o_{q}$ is the $L^{\alpha} m o$-space equipped with the equivalent norm involving oscillations in $L^{q}$. Using the JohnNirenberg inequality, we estimate ${ }^{7}$

\footnotetext{
7 We note that using Proposition 4.6, this inequality may be weakened with a growth of order $q^{\delta}$ for $\delta>0$ and maybe just some logarithmic growth on $q$. Unfortunately, this improvement does not really help to get around the (as small as we want) loss of regularity from the initial condition $\omega \in$ Lmo and the solution. We just point out that taking into account this improvement, the solution can be shown to live into a Morrey-Campanato space smaller than $L^{\alpha}$ mo with only a $\log -\log \operatorname{loss}$ of regularity. Without details, we could bound the oscillation on a ball of radius $r$ by $\frac{\log (|\log (r)|)}{|\log (r)|}$ instead of $|\log (r)|^{\delta-1}$ as we are doing here.
} 


$$
\left\|\omega_{0}\right\|_{L^{\alpha} m o_{q}} \leq C q\left\|\omega_{0}\right\|_{L^{\alpha} m o},
$$

for a universal constant $C$. This yields

$$
\operatorname{Avg}_{B}\left|\omega-\operatorname{Avg}_{B} \omega\right| \leq C q e^{\frac{2}{q} V|\ln r|^{1-\alpha}}|\ln r|^{-\alpha}\left\|\omega_{0}\right\|_{L^{\alpha} m o} .
$$

Optimizing in $q \geq 2$ (which means to chose $q=2(V+1)|\ln r|^{1-\alpha}$ ) gives

$$
\operatorname{Avg}_{B}\left|\omega-\operatorname{Avg}_{B} \omega\right| \leq C(1+V)|\ln r|^{1-\alpha}|\ln r|^{-1}\left\|\omega_{0}\right\|_{L^{\alpha} m o} .
$$

Hence

$$
|\ln (r)|^{\alpha} \operatorname{Avg}_{B}\left|\omega-\operatorname{Avg}_{B} \omega\right| \leq C(1+V)\left\|\omega_{0}\right\|_{L^{\alpha} m o} .
$$

Proof of Theorem 4.1. Using Propositions 2.9, 2.10 and Corollary 4.4, we get the following a priori estimate:

$$
\|\omega(t)\|_{L^{\alpha(t)} m o \cap L^{2}} \lesssim\left\|\omega_{0}\right\|_{L m o \cap L^{2}}\left(1+C \int_{0}^{t} \frac{1}{1-\alpha(s)}\|\omega(s)\|_{L^{\alpha(s)} m o \cap L^{2}} d s\right) .
$$

We are free to choose $\alpha(t)$ as we wish in order to get something useful out of the previous inequality. We wish to choose $\alpha$ so that $\alpha(0)=1$. However, in order that inequality (4.6) not be an empty inequality, we will need $\alpha(t)$ to decrease very sharply near $t=0$ in such a way that $\frac{1}{1-\alpha(t)}$ is integrable near $t=0$. To simplify things, we will define $\alpha(t)$ in the following way:

$$
\begin{aligned}
& \alpha(t)=1-\sqrt{t}, \quad 0 \leq t \leq \delta^{2}, \\
& \alpha(t)=1-\delta, \quad t>\delta^{2} .
\end{aligned}
$$

Note that $\alpha$ is continuous on $[0, \infty)$. Using Gronwall inequality for (4.6), we see that $\omega$ satisfies the following a priori estimate:

$$
\|\omega(t)\|_{L^{\alpha(t)} m o \cap L^{2}} \leq\left\|\omega_{0}\right\|_{L m o \cap L^{2}} \exp \left(C \gamma(t)\left\|\omega_{0}\right\|_{L m o \cap L^{2}}\right),
$$

where

$$
\gamma(t)=\int_{0}^{t} \frac{1}{1-\alpha(s)} d s= \begin{cases}2 \sqrt{t}, & t \in\left[0, \delta^{2}\right] \\ \delta+\frac{t}{\delta}, & t \in\left[\delta^{2},+\infty[.\right.\end{cases}
$$

The easy fact that $\gamma(t)$ is bounded by $2+\frac{t}{\delta}$ concludes the proof (remember that $\delta<1$ ).

\subsection{The inviscid limit when $\omega_{0} \in$ Lmo, Theorem 1.3}

In this section we will prove a sharper result on the rate of convergence in the inviscid limit of the Navier-Stokes equations in Theorem 1.1 when the initial data is taken in Lmo. Indeed, in the proof of Theorem 1.1, all we used is an a priori estimate on $u$ in BMO. However, when we take initial data in Lmo, we will be able to use a priori estimates on $L^{1-\delta} m o$ for all $\delta>0$. This fact, coupled with a sharper version of Lemma 2.7 in the $L^{\alpha} m o$ case will allow us to give a better rate than the $\varepsilon^{e^{-t}}$ from Theorem 1.1.

In particular, we will be able to prove the following theorem.

Theorem 4.5. Assume $p \in[1,2)$. Let $u_{0} \in L^{2}\left(\mathbb{R}^{2}\right)$ be a divergence free vector field such that $\omega_{0} \in$ Lmo $\cap L^{p}$. Then, for every $T>0$ and for every $\delta \in(0,1)$ there exist $C=C\left(u_{0}, \delta\right)$ and $\varepsilon_{0}=\varepsilon_{0}\left(u_{0}, T, \delta\right)$ such that

$$
\left\|u^{\varepsilon}(t)-u(t)\right\|_{L^{2}\left(\mathbb{R}^{2}\right)} \leq(C T \varepsilon)^{\frac{1}{2} e^{\beta(t)}}, \quad \forall t \in[0, T], \forall \varepsilon \leq \varepsilon_{0},
$$

with

$$
\beta(t)=\max \left(1-\delta,\left(1-\frac{e^{C_{0} t}-1}{2}\right)^{\frac{1}{\delta}}\right) .
$$


To prove this theorem we will rely upon a generalized version of the John-Nirenberg lemma in $L^{\alpha} m o$.

Proposition 4.6. Let $\alpha \in[0,1)$, then there exist $C_{1}$ and $C_{2}$ depending only upon the dimension and $0 \leq \alpha<1$ such that given any cube $Q$ in $\mathbb{R}^{n}$, any function $f \in L^{\alpha}$ mo and any $\lambda>0$,

$$
\left|\left\{x \in Q:\left|f(x)-\operatorname{Avg}_{Q} f\right|>\lambda\right\}\right| \leq C_{1} \exp \left(-\frac{C_{2}}{\|f\|_{L^{\alpha} m o}} \lambda^{\frac{1}{1-\alpha}}\right)|Q| .
$$

The proof of this proposition can be found, for example, in the paper of Caffarelli and Huang [8, Remark 2.4] and in the work of Spanne [29]. The proof is a simple adaptation of the original proof of the John-Nirenberg inequality using the Calderón-Zygmund decomposition.

Based upon this proposition, we have the following John-Nirenberg inequality (uniformly in $r \gg 1$ )

$$
\|f\|_{L^{\alpha} m o_{r}} \lesssim r^{1-\alpha}\|f\|_{L^{\alpha} m o},
$$

where $L^{\alpha} m o_{r}$ stands for the $L^{\alpha} m o$-norm with oscillations controlled in $L^{r}$. Then, one can use the proof of Lemma 2.7 to prove:

Lemma 4.7. There exists $C>0$ depending upon $\alpha \in[0,1)$ such that the following estimate holds for every $p \in$ $[2,+\infty[$ and every smooth function $f$

$$
\|f\|_{L^{p}} \leq C p^{1-\alpha}\|f\|_{L^{2} \cap L^{\alpha} m o} .
$$

Proof of Theorem 4.5. Theorem 4.5 follows from the proof of Theorem 1.1, replacing Lemma 2.7 by the last one. With the same notations, $g^{\varepsilon}(t):=\left\|U^{\varepsilon}(t)\right\|_{L^{2}}^{2}$ and

$$
T^{\varepsilon}:=\max \left\{t \leq T: \sup _{\tau \in[0, t]} g^{\varepsilon}(\tau) \leq 1\right\} .
$$

Repeating the same calculus as in the proof of Theorem 1.1 we get for every $\delta \in(0,1)$ :

$$
g^{\varepsilon}(t) \leq C \varepsilon t+\int_{0}^{t} C e^{C t^{\prime}}\left|\ln \left(g^{\varepsilon}\left(t^{\prime}\right)\right)\right|^{1-\delta} g^{\varepsilon}\left(t^{\prime}\right) d t^{\prime},
$$

with $C=C\left(\left\|u_{0}\right\|_{L^{p} \cap L m o}, \delta\right)$. Using Lemma 3.1 we infer

$$
-\left|\ln \left(g^{\varepsilon}\right)(t)\right|^{\delta}+|\ln (C t \varepsilon)|^{\delta} \leq e^{C t}-1, \quad \forall t<T^{\varepsilon} .
$$

This yields

$$
\begin{aligned}
\left|\ln \left(g^{\varepsilon}\right)(t)\right|^{\delta} & \geq|\ln (C t \varepsilon)|^{\delta}-\left(e^{C t}-1\right) \\
& \geq \beta(t)^{\delta}|\ln (C t \varepsilon)|^{\delta},
\end{aligned}
$$

with

$$
\beta(t):=\max \left(1-\delta,\left(2-e^{C t}\right)^{\frac{1}{\delta}}\right) \leq\left(1-\frac{e^{C t}-1}{|\ln (C t \varepsilon)|^{\delta}}\right)^{\frac{1}{\delta}} .
$$

The previous inequality holds for $\varepsilon \leq \varepsilon_{0}$ where $\varepsilon_{0}=\varepsilon_{0}\left(u_{0}, T, \delta\right)$ is chosen so that:

$$
\left|\ln \left(C T \varepsilon_{0}\right)\right| \geq 1 \quad \text { and } \quad\left(1-\frac{e^{C T}-1}{\left|\ln \left(C T \varepsilon_{0}\right)\right|}\right) \geq(1-\delta)^{\delta} .
$$

Then we conclude by reproducing the same reasoning as for Theorem 1.1, with these slight modifications. 


\section{Uniform estimates for solutions of Navier-Stokes equation with a vorticity in $L^{\alpha} m o$ for $\alpha>1$}

In this section, we aim to describe more results when we assume that the vorticity is more regular, and more precisely when $\omega_{0} \in L^{\alpha} m o$ for some $\alpha>1$.

Remark 5.1. When the velocity $u$ is associated to an $L^{\alpha} m o$ vorticity for some $\alpha>1$ by the Biot-Savart law (2.1), the combination of Lemma 2.5 and Proposition 2.6 yields that $u$ is Lipschitz.

We first aim to prove a slight improvement of results in [5], about composition in $L^{\alpha} m o$-spaces by a bi-Lipschitz measure-preserving map.

\subsection{Composition in $L^{\alpha}$ mo by a bi-Lipschitz map}

Theorem 5.2. In $\mathbb{R}^{d}$, there exists a constant $c:=c(d)$ such that for every function $f \in \mathrm{BMO}$ and every measurepreserving bi-Lipschitz homeomorphism $\phi$, we have

$$
\|f \circ \phi\|_{\mathrm{BMO}} \leq\|f\|_{\mathrm{BMO}}\left[1+c \log \left(K_{\phi}\right)\right],
$$

where

$$
K(\phi)=K_{\phi}:=\sup _{x \neq y} \max \left(\frac{|\phi(x)-\phi(y)|}{|x-y|}, \frac{|x-y|}{|\phi(x)-\phi(y)|}\right) \geq 1 .
$$

Remark 5.3. Let us first point out that this property of BMO space is not invariant by changing with an equivalent norm. So the precise statement should be: there exists a norm such that Theorem 5.2 holds for BMO equipped with it.

Remark 5.4. In [5], such a result was already obtained with a control by (on the r.h.s.) $c_{1}\left[1+c \log \left(K_{\phi}\right)\right]$ with an implicit constant $c_{1}>1$. The aim here is to improve by proving that $c_{1}$ may be chosen equal to 1 , which brings an important improvement for when the map $\phi$ converges to the identity or any isometry (which is equivalent to $K_{\phi}$ converges to 1). This improvement will be very important for our purpose in the next subsections, as we will see.

Proof of Theorem 5.2. We will consider the norm of $\mathrm{BMO}_{2}$ based on $L^{2}$-oscillation. If $K_{\phi} \geq 2$ then the desired result was already obtained in [5] since then

$$
1+c \log \left(K_{\phi}\right) \simeq \log \left(K_{\phi}\right) .
$$

So let us focus on the more interesting case, when $K_{\phi} \in[1,2]$. Consider such a function $f \in$ BMO and map $\phi$. Fix a ball $B=B\left(x_{0}, r\right)$ and look for an estimate of the oscillation

$$
\operatorname{Osc}(f \circ \phi, B):=\left(\operatorname{Avg}_{B}\left|f \circ \phi(x)-\operatorname{Avg}_{B} f \circ \phi\right|^{2}\right)^{\frac{1}{2}} .
$$

Then, it is well-known that

$$
\operatorname{Osc}(f \circ \phi, B)=\inf _{C \in \mathbb{R}}\left(\operatorname{Avg}_{B}|f \circ \phi(x)-C|^{2}\right)^{\frac{1}{2}}
$$

and so in particular

$$
\operatorname{Osc}(f \circ \phi, B) \leq\left(\operatorname{Avg}_{B}\left|f \circ \phi(x)-\operatorname{Avg}_{K_{\phi} \tilde{B}} f\right|^{2}\right)^{\frac{1}{2}},
$$

where $\tilde{B}:=B\left(\phi\left(x_{0}\right), r\right)$ and $K_{\phi} \tilde{B}$ is the dilated ball. Using the measure preserving property and the fact that $\phi(B) \subset K_{\phi} \tilde{B}$, it comes

$$
\begin{aligned}
\operatorname{Osc}(f \circ \phi, B) & \leq\left(\operatorname{Avg}_{\phi(B)}\left|f-\operatorname{Avg}_{K_{\phi} \tilde{B}} f\right|^{2}\right)^{\frac{1}{2}} \\
& \leq\left(K_{\phi}\right)^{d / 2}\left(\operatorname{Avg}_{K_{\phi} \tilde{B}}\left|f-\operatorname{Avg}_{K_{\phi} \tilde{B}} f\right|^{2}\right)^{\frac{1}{2}} \\
& \leq K_{\phi}^{d / 2}\|f\|_{\text {BMO }} .
\end{aligned}
$$


Since $K_{\phi} \in[1,2]$, we have

$$
K_{\phi}^{d / 2}=\left(1+K_{\phi}-1\right)^{d / 2} \leq 1+c_{1}\left(K_{\phi}-1\right) \leq 1+c_{2} \log \left(K_{\phi}\right),
$$

for some numerical constants $c_{1}, c_{2}$ only depending on the dimension $d$. We conclude to the desired estimate: uniformly with respect to the ball $B$

$$
\operatorname{Osc}(f \circ \phi, B) \leq\left[1+c \log \left(K_{\phi}\right)\right]\|f\|_{\mathrm{BMO}} .
$$

We can also produce a similar reasoning for the $L^{\alpha}$ mo spaces:

Theorem 5.5. In $\mathbb{R}^{d}$ with $\alpha>1, p \in(1,2]$, there exists a constant $c:=c(d, \alpha, p)$ such that for every function $f \in$ $L^{\alpha} m o \cap L^{p}$ and every measure-preserving bi-Lipschitz homeomorphism $\phi$, we have

$$
\|f \circ \phi\|_{L^{\alpha} m o \cap L^{p}} \leq\|f\|_{L^{\alpha} m o \cap L^{p}}\left[1+c \log \left(K_{\phi}\right)\right]^{\alpha},
$$

where

$$
K(\phi)=K_{\phi}:=\sup _{x \neq y} \max \left(\frac{|\phi(x)-\phi(y)|}{|x-y|}, \frac{|x-y|}{|\phi(x)-\phi(y)|}\right) \geq 1 .
$$

Moreover, $\alpha \rightarrow c(d, \alpha, p)$ can be chosen increasing on $\mathbb{R}^{+}$.

The importance of the result is the behavior for $\phi$ almost an isometry, which means $K_{\phi}$ almost equal to 1 .

Proof. Since the case of the logarithmic growth for $K_{\phi} \geq 2$ was already studied in [5], we only focus on the case $K_{\phi} \in[1,2]$. We first describe the norm we will consider on $L^{\alpha} m o \cap L^{p}$ :

$$
\|f\|_{L^{\alpha} m o \cap L^{p}}=\|f\|_{L^{\alpha} m o}+\|f\|_{L^{p}},
$$

where the $\overline{L^{\alpha} m o}$-part is the homogeneous part, obtained by considering $L^{1}$-oscillations and more precisely:

$$
\|f\|_{L^{\alpha} m o}:=\sup _{0<r \leq \frac{1}{2}}|\ln r|^{\alpha} \inf _{c}\left(\operatorname{Avg}_{B}|f-c|\right) .
$$

We know that this norm is equivalent to the above defined norm for $L^{\alpha} m o \cap L^{p}$. So let us work with this norm and write

$$
\operatorname{Osc}(f, B):=\inf _{c}\left(\operatorname{Avg}_{B}|f-c|\right) .
$$

First $\Phi$ preserves the measure so $\|f \circ \phi\|_{L^{p}}=\|f\|_{L^{p}}$. Let us consider the same notations as in the previous proof. So we fix a ball $B=B\left(x_{0}, r\right)$ of radius $r \leq \frac{1}{2}$ and a constant $c$. If $K_{\phi} r \leq \frac{1}{2}$ then we just repeat the previous reasoning and we get

$$
\begin{aligned}
\operatorname{Osc}(f \circ \phi, B) & \leq\left(K_{\phi}\right)^{d} \operatorname{Osc}\left(f, K_{\phi} \tilde{B}\right) \\
& \leq\left(K_{\phi}\right)^{d}\left|\log \left(K_{\phi} r\right)\right|^{-\alpha}\|f\|_{L^{\alpha} m o} \leq\left(K_{\phi}\right)^{d}\left(1+\log \left(K_{\phi}\right)\right)^{\alpha}|\log (r)|^{-\alpha}\|f\|_{L^{\alpha} m o},
\end{aligned}
$$

where we used that

$$
\frac{|\log (r)|}{|\log (r)|-\log \left(K_{\phi}\right)}=1+\frac{\log \left(K_{\phi}\right)}{|\log (r)|-\log \left(K_{\phi}\right)} \leq 1+\frac{\log \left(K_{\phi}\right)}{\log (2)} .
$$

Since $K_{\phi} \in[1,2]$, we have (since $\alpha \geq 1$ )

$$
\left(K_{\phi}\right)^{d}\left(1+\log \left(K_{\phi}\right)\right)^{\alpha} \leq\left(1+c \log \left(K_{\phi}\right)\right)
$$

for some (large enough) numerical constant $c \gg d+\alpha$. We then conclude to

$$
\operatorname{Osc}(f \circ \phi, B) \leq\left(1+c \log \left(K_{\phi}\right)\right)|\log (r)|^{-\alpha}\|f\|_{L^{\alpha} m o} .
$$


If $K_{\phi} r \geq \frac{1}{2}$ (which means that $r \geq \frac{1}{4}$ ) then we know that

$$
\left|\operatorname{Avg}_{\phi(B)}\right| f\left|-\operatorname{Avg}_{\tilde{B}}\right| f|| \lesssim\left(K_{\phi}-1\right)\|f\|_{L^{\infty}}
$$

since $\phi(B) \subset K_{\phi} \tilde{B}$ and $K_{\phi}^{-1} \tilde{B} \subset \phi(B)$ so that

$$
|\phi(B) \backslash \tilde{B}|+|\tilde{B} \backslash \phi(B)| \lesssim\left(K_{\phi}-1\right) r^{d} .
$$

Due to Lemma 2.5, we deduce that

$$
\left|\operatorname{Avg}_{\phi(B)}\right| f\left|-\operatorname{Avg}_{\tilde{B}}\right| f|| \lesssim\left(K_{\phi}-1\right)\|f\|_{L^{\alpha} m o} .
$$

Consequently, it comes

$$
\begin{aligned}
\operatorname{Osc}(f \circ \phi, B) & =\inf _{c}\left(\operatorname{Avg}_{\phi(B)}|f-c| d x\right) \\
& \leq \operatorname{Osc}(f, \tilde{B})+C\left(K_{\phi}-1\right)\|f\|_{L^{\alpha} m o} \\
& \leq|\log (r)|^{-\alpha}\|f\|_{L^{\alpha} m o}+C\left(K_{\phi}-1\right)\|f\|_{L^{\alpha} m o},
\end{aligned}
$$

where $C$ denotes here a universal constant and may vary from line to another line. Since $r \geq \frac{1}{4}$ we get

$$
\operatorname{Osc}(f \circ \phi, B) \leq|\log (r)|^{-\alpha}\left(\|f\|_{L^{\alpha} m o}+C\left(K_{\phi}-1\right)\|f\|_{L^{\alpha} m o}\right) .
$$

Finally, we also obtain that

$$
\|f \circ \phi\|_{L^{p} \cap L^{\alpha} m o} \leq\left(1+C\left(K_{\phi}-1\right)+c \log \left(K_{\phi}\right)\right)\|f\|_{L^{p} \cap L^{\alpha} m o},
$$

and we conclude since for $K_{\phi} \in[1,2],\left(K_{\phi}-1\right) \lesssim \log \left(K_{\phi}\right)$.

\subsection{Uniform estimates for discretized solutions of the $2 D$ Navier-Stokes equation}

In this paragraph the small parameter $\varepsilon$ in Navier-Stokes equation (1.1) is fixed. For simplicity we drop the in$\operatorname{dex} \varepsilon$. We aim to discretize this equation, using the so-called Trotter's formula to combine the two phenomenons: the transport part and the diffusion part.

Let $T>0$ to be chosen later. For every $n \in \mathbb{N}^{*}$ one denotes

$$
T_{i}^{n}=i \frac{T}{n}, \quad i=0, \ldots, n .
$$

We consider the following scheme: for every $n \in \mathbb{N}^{*}$ one constructs $u^{n}$ as follows:

- $u^{n}$ belongs to $C\left([0, T], L^{2}\right)$ with the initial condition

$$
u^{n}(0)=u_{0},
$$

- if $t \in\left[T_{i}^{n}, T_{i+1}^{n}\right]$ with $i \in 2 \mathbb{N}$ and $i<n$

$$
\left\{\partial_{t} u^{n}-2 \varepsilon \Delta u^{n}=0, \quad x \in \mathbb{R}^{2}, t>0,\right.
$$

- if $t \in\left[T_{i}^{n}, T_{i+1}^{n}\right]$ with $i \in 2 \mathbb{N}+1$ and $i<n$

$$
\left\{\begin{array}{l}
\partial_{t} u^{n}+2 u^{n} \cdot \nabla u^{n}+2 \nabla P^{n}=0, \quad x \in \mathbb{R}^{2}, t>0, \\
\nabla \cdot u^{n}=0 .
\end{array}\right.
$$

Let us note that $u^{n}$ exists and it is smooth. In fact, the first step $(i=0)$ preserves the divergence free condition and regularizes the solution and so that $u^{n}\left(T_{1}^{n}\right) \in H^{\infty}\left(\mathbb{R}^{2}\right)$. By the classical result of Kato, the Euler system (5.1) has a unique solution on $\left[T_{1}^{n}, T_{2}^{n}\right]$ which belongs to $H^{\infty}$. And then we iterate the same argument to get a (unique) piecewise smooth solution $u^{n}$ on $(0, T]$.

Let us now give a more convenient form of the different systems (5.1) and (5.2), in terms of the vorticity $\omega^{n}:=$ $\operatorname{curl}\left(u^{n}\right)$. 
The system (5.1) can be exactly solved by the heat semigroup (since it preserves the divergence free condition and commutes with the curl-operator), we may rewrite (5.1) as follows: for $t \in\left[T_{i}^{n}, T_{i+1}^{n}\right]$ with $i \in 2 \mathbb{N}$ and $i<n$

$$
\omega^{n}(t)=e^{2 \varepsilon\left(t-T_{i}^{n}\right) \Delta} \omega^{n}\left(T_{i}^{n}\right)
$$

The system (5.2) may also be written on the vorticity as follows: for $t \in\left[T_{i}^{n}, T_{i+1}^{n}\right]$ with $i \in 2 \mathbb{N}+1$ and $i<n$

$$
\left\{\partial_{t} \omega^{n}+2 u^{n} \cdot \nabla \omega^{n}=0, \quad x \in \mathbb{R}^{2}, t>0,\right.
$$

supplemented with the Biot-Savart law:

$$
u^{n}=K * \omega^{n}, \quad \text { with } K(x)=\frac{x^{\perp}}{2 \pi|x|^{2}} .
$$

As a consequence, we know that $\omega^{n}(t)=\omega^{n}\left(T_{i}^{n}\right) \circ \phi_{t-T_{i}^{n}}^{-1}$ where $\phi_{t-T_{i}^{n}}$ is the flow corresponding to the vector-field $u^{n}$.

Then, our aim is now to prove that the family $\left(u^{n}\right)_{n}$ above is uniformly bounded on the interval $[0, T]$, as soon as $T$ is small enough (depending of the initial vorticity). More precisely, one proves the following with the notation $\mathcal{B}_{p, \alpha}:=L^{\alpha} m o \cap L^{p}:$

Proposition 5.6. Let $p \in[1,2)$ and $\alpha>1, \omega_{0} \in \mathcal{B}_{p, \alpha}$. There exists $T \approx \frac{1}{\left\|\omega_{0}\right\|_{\mathcal{B}_{p, \alpha}}}$ such that the family $\left(\omega^{n}\right)_{n \in \mathbb{N}^{*}}$ is uniformly bounded in $\mathcal{B}_{p, \alpha}$. More precisely,

$$
\left\|u^{n}\right\|_{L^{\infty}\left([0, T], L^{2}\right)}+\left\|u^{n}\right\|_{L^{\infty}([0, T], \operatorname{Lip})}+\left\|\omega^{n}\right\|_{L^{\infty}\left([0, T], \mathcal{B}_{p, \alpha}\right)} \leq 2\left\|\omega_{0}\right\|_{\mathcal{B}_{p, \alpha}} .
$$

Proof. For $h:=\frac{T}{n}$ with $n$ large enough such that $h \ll T^{-1}$, consider the discrete solution $\omega^{n}$ given by (5.3) and (5.4). We write $X_{0}:=\|\omega(0)\|_{\mathcal{B}_{p, \alpha}}$ and for $k \in\{1, \ldots, n\}$

$$
X_{k}=\sup _{[0, k h]}\left\|\omega^{n}(t)\right\|_{\mathcal{B}_{p, \alpha}} .
$$

If $k \in 2 \mathbb{N}$ and $k<n-1$ then $\omega^{n}$ on $[k h,(k+1) h]$ is given by (5.3) and so by Remark 2.4

$$
X_{k+1} \leq X_{k}
$$

If $k \in 2 \mathbb{N}+1$ and $k<n-1$ then $\omega^{n}$ on $\left[T_{k}^{n}, T_{k+1}^{n}\right]$ is given by (5.4) and so from Proposition 2.9, Proposition 2.11 and Theorem 5.5, we have

$$
X_{k+1} \leq X_{k} \exp \left(\mu X_{k} h\right),
$$

for some numerical constant $\mu$ (here we have used that $(1+x)^{\alpha} \leq \exp (\alpha x)$ for $x \geq 0$ ).

As a consequence, the sequence $\left(X_{k}\right)_{k}$ satisfies the following growth condition: for every $k \in\{1, \ldots, n\}$

$$
X_{k} \leq X_{k-1} \exp \left(\mu X_{k-1} h\right)
$$

where $\mu$ is a universal constant.

By iteration, we deduce that

$$
X_{k} \leq X_{0} \exp \left(\mu\left(X_{0}+\cdots+X_{k-1}\right) h\right) .
$$

Let us assume that $X_{j} \leq 2 X_{0}$ for every $j<k$ then by (5.6) we deduce

$$
X_{k} \leq X_{0} \exp \left(\mu T 2 X_{0}\right) \text {. }
$$

One chooses $T$ such that

$$
\exp \left(\mu T 2 X_{0}\right)=2,
$$

to conclude

$$
X_{k} \leq 2 X_{0} .
$$

By iterating this reasoning, it comes that (5.7) holds for every $k \leq n$ which combined with Propositions 2.9 and 2.11 gives the desired estimate. 


\subsection{Convergence to a solution of the Navier-Stokes equation}

According to Proposition 5.6, there exists a subsequence $\left(u^{n}\right)_{n} *$-weak converging to $u \in L^{\infty}([0, T]$, Lip) and such that $\omega^{n} *$-weakly converges to $\omega \in L^{\infty}\left([0, T], \mathcal{B}_{p, \alpha}\right)$.

Proposition 5.7. The limit $(u, \omega)$ is a solution of $2 D$ Navier-Stokes equation

$$
\partial_{t} \omega+u \cdot \nabla \omega-\varepsilon \Delta \omega=0
$$

and satisfies uniform estimates with respect to $\varepsilon>0$ :

$$
\|u\|_{L^{\infty}([0, T], \text { Lip })}+\|\omega\|_{L^{\infty}\left([0, T], \mathcal{B}_{p, \alpha}\right)} \leq 2\left\|\omega_{0}\right\|_{\mathcal{B}_{p, \alpha}},
$$

where $T=T\left(\omega_{0}\right)$ is given in Proposition 5.6.

This also proves Theorem 1.10 for the solution of Navier-Stokes equations. We let the reader to check that for the case of fractional Navier-Stokes is exactly the same, since for $\sigma \in(0,1)$ the heat kernel of $e^{-t(-\Delta)^{\sigma}}$ is given by a non-negative $L^{1}$-normalized function (see $[1,16]$ ).

Proof. The corresponding estimates on $u$ and $\omega$ directly follow from the uniform estimates of Proposition 5.6. So it remains us to check that $(u, \omega)$ is a solution of 2D Navier-Stokes equation.

Let $\phi \in C^{\infty}\left(\left[0, T\left[\times \mathbb{R}^{2}\right)\right.\right.$ compactly supported. For every $h$ small enough, we have

$$
\sum_{i=0}^{n / 2} \int_{T_{2 i}^{n}}^{T_{2 i+1}^{n}}\left\langle\partial_{t} \omega^{n}-2 \varepsilon \Delta \omega^{n}, \phi\right\rangle d s+\int_{T_{2 i+1}^{n}}^{T_{2 i+2}^{n}}\left\langle\partial_{t} \omega^{n}+2 u^{n} \cdot \nabla \omega^{n}, \phi\right\rangle d s=0,
$$

since each term is equal to 0 .

Using the initial condition on the interval $\left[T_{2 i}^{n}, T_{2 i+1}^{n}\right]$ and the vanishing divergence of $u^{n}$, it comes

$$
\int_{T_{2 i+1}^{n}}^{T_{2 i+2}^{n}}\left\langle\partial_{t} \omega^{n}+2 u^{n} \cdot \nabla \omega^{n}, \phi\right\rangle d s=-\int_{T_{2 i+1}^{n}}^{T_{2 i+2}^{n}}\left\langle\omega^{n}, \partial_{t} \phi+2 u^{n} \cdot \nabla \phi\right\rangle d s+\left[\left\langle\omega^{n}, \phi\right\rangle\right]_{T_{2 i+1}^{n}}^{T_{2 i+2}^{n}}
$$

and

$$
\int_{T_{2 i}^{n}}^{T_{2 i+1}^{n}}\left\langle\partial_{t} \omega^{n}-2 \varepsilon \Delta \omega^{n}, \phi\right\rangle d s=-\int_{T_{2 i}^{n}}^{T_{2 i+1}^{n}}\left\langle\omega^{n}, \partial_{t} \phi+2 \varepsilon \Delta \phi\right\rangle d s+\left[\left\langle\omega^{n}, \phi\right\rangle\right]_{T_{2 i}^{n}}^{T_{2 i+1}^{n}} .
$$

So by summing over $i,(5.8)$ becomes

$$
\left\langle\omega_{0}, \phi(0)\right\rangle+\int_{0}^{T}\left\langle\omega^{n}, \partial_{t} \phi\right| d s-\sum_{i=0}^{n / 2} \int_{T_{2 i+1}^{n}}^{T_{2 i+2}^{n}}\left\langle\omega^{n}, 2 u^{n} \cdot \nabla \phi\right\rangle d s+2 \varepsilon \int_{T_{2 i}^{n}}^{T_{2 i+1}^{n}}\left\langle\omega^{n}, \Delta \phi\right\rangle d s .
$$

The family $\partial_{t} u_{n}$ is bounded in $L^{\infty}\left([0, T], H^{-2}\right)$. Using Ascoli-Arzelà and Rellich theorems we get that (up to extracting a subsequence) we may assume that the convergence of $u^{n}$ to $u$ is strong in $L^{2}([0, T] \times K)$ for every compact $K \subset \mathbb{R}^{2}$.

Due to the weak convergence of $\omega_{n}$, we get

$$
\int_{0}^{T}\left\langle\omega^{n}, \partial_{t} \phi\right\rangle d s \underset{h \rightarrow 0}{\longrightarrow} \int_{0}^{T}\left\langle\omega, \partial_{t} \phi\right\rangle d s .
$$

We have (using the notation of Lemma 5.8) 


$$
\sum_{i=0}^{n / 2} \int_{(2 i+1) h}^{2(i+1) h}\left\langle\omega^{n}, \Delta \phi\right\rangle d s=\int_{0}^{T}\left\langle\omega^{n}(t),(\Delta \phi(t))_{h}\right\rangle d t
$$

Since $\omega^{n}=\operatorname{curl}\left(u^{n}\right)$, by integration by parts in the physical space we have

$$
\sum_{i=0}^{n / 2} \int_{(2 i+1) h}^{2(i+1) h}\left\langle\omega^{n}, \Delta \phi\right\rangle d s=\int_{0}^{T}\left\langle u^{n}(t),\left(\operatorname{curl}^{*} \Delta \phi(t)\right)_{h}\right\rangle d t .
$$

Then using that $\left(u_{n}\right)_{n}$ strongly converges into $L^{2}([0, T] \times K)$ (where $K$ is a compact including the space-support of $\phi)$ and according to Lemma $5.8\left(\operatorname{curl}^{*} \Delta \phi(t)\right)_{h}$ weakly converges in $L^{2}$ then we conclude that

$$
\begin{aligned}
\lim _{h \rightarrow 0} \sum_{i=0}^{n / 2} \int_{(2 i+1) h}^{2(i+1) h}\left\langle\omega^{n}, \Delta \phi\right\rangle d s & =\frac{1}{2} \int_{0}^{T}\left\langle u(t), \operatorname{curl}^{*} \Delta \phi(t)\right\rangle d t \\
& =\frac{1}{2} \int_{0}^{T}\langle\omega(t), \Delta \phi(t)\rangle d t .
\end{aligned}
$$

For the third term, we decompose $u^{n}=\tilde{u}+\left(u^{n}-\tilde{u}\right)$ with a smooth function $\tilde{u}$ so that

$$
\left\langle\omega^{n}, 2 u^{n} \cdot \nabla \phi\right\rangle=\left\langle\omega^{n}, 2 \tilde{u} \cdot \nabla \phi\right\rangle+\left\langle\omega^{n}, 2\left(u^{n}-\tilde{u}\right) \cdot \nabla \phi\right\rangle .
$$

As previously, using Lemma 5.8, we have

$$
\sum_{i=0}^{n / 2} \int_{2 i h}^{(2 i+1) h}\left\langle\omega^{n}, 2 \tilde{u} \cdot \nabla \phi\right\rangle d s \underset{h \rightarrow 0}{\longrightarrow} \int_{0}^{T}\langle\omega, \tilde{u} \cdot \nabla \phi\rangle d s .
$$

Moreover,

$$
\begin{aligned}
\left|\sum_{i=0}^{n / 2} \int_{2 i h}^{(2 i+1) h}\left\langle\omega^{n}, 2\left(u^{n}-\tilde{u}\right) \cdot \nabla \phi\right\rangle d s\right| & \lesssim \sum_{i=0}^{n / 2} \int_{2 i h}^{(2 i+1) h}\left\|\omega^{n}\right\|_{L^{2}}\left\|u^{n}-\tilde{u}\right\|_{L^{2}}\|\nabla \phi\|_{L^{\infty}} d s \\
& \lesssim T\left\|\omega^{n}\right\|_{L^{2}\left([0, T], L^{2}\right)}\left\|\tilde{u}-u^{n}\right\|_{L^{2}\left([0, T], L^{2}(\operatorname{Supp}(\phi))\right)} \\
& \lesssim\left\|\tilde{u}-u^{n}\right\|_{L^{2}\left([0, T], L^{2}(\operatorname{Supp}(\phi))\right)}
\end{aligned}
$$

So finally, using the local strong convergence in $L^{2}\left([0, T], L^{2}\right)$ of $\left(u^{n}\right)_{n}$ we have

$$
\underset{h \rightarrow 0}{\limsup }\left|\sum_{i=0}^{n / 2} \int_{2 i h}^{(2 i+1) h}\left\langle\omega^{n}, 2 u^{n} \cdot \nabla \phi\right\rangle d s-\int_{0}^{T}\langle\omega, 2 u \cdot \nabla \phi\rangle\right| \lesssim \inf _{\tilde{u} \in C_{0}^{\infty}}\|\tilde{u}-u\|_{L^{2}\left([0, T], L^{2}\right)}=0 .
$$

So taking the limit when $h \rightarrow 0$ in (5.9) yields

$$
\left\langle\omega_{0}, \phi(0)\right\rangle+\int_{0}^{T}\left\langle\omega, \partial_{t} \phi\right\rangle d s-\int_{0}^{T}\langle\omega, u \cdot \nabla \phi\rangle d s-\varepsilon \int_{0}^{T}\langle\omega, \Delta \phi\rangle d s,
$$

which by integrations by parts in time gives (in a distributional sense)

$$
\int_{0}^{T}\left\langle\partial_{t} \omega+u \cdot \nabla \omega-\varepsilon \Delta \omega, \phi\right\rangle d s=0 .
$$


This last equality holds for every compactly supported smooth function $\phi \in C_{0}^{\infty}\left([0, T) \times \mathbb{R}^{2}\right)$, so we deduce that $(\omega, u)$ is a solution of Navier-Stokes equation. By uniqueness of solution, $(\omega, u)$ is the solution of Navier-Stokes equation and satisfies the uniform estimates.

Lemma 5.8. With the previous notations, let $f$ be a compactly supported smooth function on $[0, T] \times \mathbb{R}^{2}$, then

$$
f_{h}:=\sum_{i=0}^{N / 2} \mathbf{1}_{[(2 i+1) h, 2(i+1) h]}(t) f
$$

weakly converges in $L^{2}\left([0, T], L^{2}\right)$ to $\frac{1}{2} f$ when h goes to 0 .

Proof. Since $\left(f_{h}\right)_{h>0}$ is uniformly bounded in $L^{2}\left([0, T], L^{2}\right)$, it suffices us to check that for every smooth function $g$

$$
\lim _{h \rightarrow 0} \iint f_{h}(t, x) g(t, x) d t d x=\frac{1}{2} \iint f(t, x) g(t, x) d t d x .
$$

So let us fix such a function $g$ and set

$$
\widetilde{f}_{h}:=\sum_{i=0}^{N / 2} \mathbf{1}_{[2 i h,(2 i+1) h]}(t) f
$$

such that $f=f_{h}+\widetilde{f}_{h}$. However, it is clear (by a first order expansion in the time variable) that

$$
\left|\int_{2 i h}^{(2 i+1) h} \int f(t, x) g(t, x) d x d t-\int_{(2 i+1) h}^{2(i+1) h} \int f(t, x) g(t, x) d x d t\right| \leq\left\|\partial_{t}(f g)\right\|_{L^{\infty}} h^{2} .
$$

So summing over $i$ yields

$$
\left|\iint f_{h}(t, x) g(t, x) d t d x-\iint \widetilde{f}_{h}(t, x) g(t, x) d t d x\right| \lesssim h
$$

and so

$$
\lim _{h \rightarrow 0}\left|\iint f_{h}(t, x) g(t, x) d t d x-\iint \tilde{f}_{h}(t, x) g(t, x) d t d x\right|=0 .
$$

We conclude with the equality $f=f_{h}+\widetilde{f}_{h}$ which gives

$$
\frac{1}{2} f-f_{h}=\frac{1}{2}\left(f_{h}-\tilde{f}_{h}\right)
$$

\section{Conflict of interest statement}

For the publication entitled "On the inviscid limit of the 2D Navier-Stokes equations with vorticity belonging to BMO-type spaces", the three authors (Frédéric Bernicot, Tarek Elgindi and Sahbi Keraani) declare that there is no conflicts of interest associated with this publication.

\section{References}

[1] R.M. Blumenthal, R.K. Getoor, Some theorems on stable processes, Trans. Amer. Math. Soc. 95 (1960) 263-273.

[2] H. Bahouri, J.-Y. Chemin, R. Danchin, Fourier Analysis and Nonlinear Partial Differential Equations, Grundlehren Math. Wiss., vol. 343, 2011.

[3] F. Bernicot, J.M. Martell, Self-improving properties for abstract Poincaré type inequalities, Trans. Amer. Math. Soc. (2015), http://dx.doi.org/ 10.1090/S0002-9947-2014-06315-0, in press.

[4] F. Bernicot, T. Hmidi, On the global well-posedness for Euler equations with unbounded vorticity, arXiv:1303.6151.

[5] F. Bernicot, S. Keraani, Sharp constants for composition with a measure-preserving map, Math. Res. Lett. 21 (2014) $937-952$. 
[6] F. Bernicot, S. Keraani, On the global well-posedness of the 2D Euler equations for a large class of Yudovich type data, Ann. Sci. Éc. Norm. Supér. (4) 47 (3) (2014) 559-576.

[7] A.L. Bertozzi, A.J. Majda, Vorticity and Incompressible Flow, Cambridge Texts Appl. Math., vol. 27, Cambridge University Press, Cambridge, 2002.

[8] L.A. Caffarelli, Q. Huang, Estimates in the generalized Campanato-John-Nirenberg spaces for fully nonlinear elliptic equations, Duke Math. J. 118 (1) (2003) $1-17$.

[9] D. Chae, Weak solutions of 2-D Euler equations with initial vorticity in L(log L), J. Differ. Equ. 103 (1993) $323-337$.

[10] J.-Y. Chemin, Perfect Incompressible Fluids, The Clarendon Press, Oxford University Press, New York, 1998.

[11] J.-Y. Chemin, A remark on the inviscid limit for two-dimensional incompressible fluids, Commun. Partial Differ. Equ. 21 (11-12) (1996) 1771-1779 (in English, French summary).

[12] Q. Chen, C. Miao, X. Zheng, The bi-dimensional Euler equations in bmo-type space, arXiv:1311.0934.

[13] E. Cozzi, J. Kelliher, Vanishing viscosity in the plane for vorticity in borderline spaces of Besov type, J. Differ. Equ. 235 (2) (2007) $647-657$.

[14] J.-M. Delort, Existence de nappes de tourbillon en dimension deux, J. Am. Math. Soc. 4 (1991) 553-586.

[15] R. DiPerna, A. Madja, Concentrations in regularization for 2D incompressible flow, Commun. Pure Appl. Math. 40 (1987) $301-345$.

[16] A. Grigor'yan, J. Hu, K.-S. Lau, Heat kernels on metric measure spaces, in: Proceedings of AFRT2012, Geometry and Analysis of Fractals, 2014, pp. 147-208.

[17] M.C. Lopes Filho, H.J. Nussenzveig Lopes, Z. Xin, Existence of vortex sheets with reflection symmetry in two space dimensions, Arch. Ration. Mech. Anal. 158 (3) (2001) 235-257.

[18] B. Franchi, C. Pérez, R.L. Wheeden, Self-improving properties of John-Nirenberg and Poincaré inequalities on space of homogeneous type, J. Funct. Anal. 153 (1) (1998) 108-146.

[19] H. Fujita, T. Kato, On the nonstationary Navier-Stokes system, Rend. Semin. Mat. Univ. Padova 32 (1962) $243-260$.

[20] P. Gérard, Résultats récents sur les fluides parfaits incompressibles bidimensionnels, d'après J.-Y. Chemin et J.-M. Delort, in: Séminire Bourbaki, 1991/1992, No. 757, Astérisque 206 (1992) 411-444.

[21] Y. Giga, T. Miyakawa, H. Osada, 2D Navier-Stokes flow with measures as initial vorticity, Arch. Ration. Mech. Anal. 104 (1988) $223-250$.

[22] L. Grafakos, Classical and Modern Fourier Analysis, Prentice Hall, New York, 2006.

[23] J. Kelliher, The inviscid limit for two-dimensional incompressible fluids with unbounded vorticity, Math. Res. Lett. 11 (4) (2004) 519-528.

[24] J. Leray, Sur le mouvement d'un liquide visqueux remplissant l'espace, Acta Math. 63 (1934) 193-248.

[25] P.-L. Lions, Mathematical Topics in Fluid Mechanics, vol. 1, The Clarendon Press, Oxford University Press, New York, 1996.

[26] N. Masmoudi, Remarks about the inviscid limit of the Navier-Stokes system, Commun. Math. Phys. 270 (3) (2007) $777-788$.

[27] J. Pfetre, On convolution operators leaving $L^{p, \lambda}$ spaces invariant, Ann. Mat. Pura Appl. 72 (1966) 295-304.

[28] P. Serfati, Structures holomorphes à faible régularité spatiale en mécanique des fluides, J. Math. Pures Appl. 74 (1995) $95-104$.

[29] S. Spanne, Some function spaces defined using the mean oscillation over cubes, Ann. Sc. Norm. Super. Pisa 19 (1965) $593-608$.

[30] Y. Taniuchi, Uniformly local $L^{p}$ estimate for $2 \mathrm{D}$ vorticity equation and its application to Euler equations with initial vorticity in BMO, Commun. Math. Phys. 248 (2004) 169-186.

[31] M. Vishik, Incompressible flows of an ideal fluid with vorticity in borderline spaces of Besov type, Ann. Sci. Éc. Norm. Supér. (4) 32 (6) (1999) 769-812 (in English, French summary).

[32] Y. Yudovich, Nonstationary flow of an ideal incompressible liquid, Zh. Vychisl. Mat. 3 (1963) 1032-1066.

[33] Y. Yudovich, Uniqueness theorem for the basic nonstationary problem in the dynamics of an ideal incompressible fluid, Math. Res. Lett. 2 (1995) 27-38. 